\title{
Positive and Nondecreasing Solutions to an $m$-Point Boundary Value Problem for Nonlinear Fractional Differential Equation
}

\author{
I. J. Cabrera, J. Harjani, and K. B. Sadarangani \\ Departamento de Matemáticas, Universidad de Las Palmas de Gran Canaria, Campus de Tafira Baja, \\ 35017 Las Palmas de Gran Canaria, Spain \\ Correspondence should be addressed to K. B. Sadarangani, ksadaran@dma.ulpgc.es \\ Received 30 September 2011; Accepted 15 November 2011 \\ Academic Editor: Shaher M. Momani
}

Copyright (C) 2012 I. J. Cabrera et al. This is an open access article distributed under the Creative Commons Attribution License, which permits unrestricted use, distribution, and reproduction in any medium, provided the original work is properly cited.

\begin{abstract}
We are concerned with the existence and uniqueness of a positive and nondecreasing solution for the following nonlinear fractional $m$-point boundary value problem: $D_{0^{+}}^{\alpha} u(t)+f(t, u(t))=0,0<$ $t<1,2<\alpha \leq 3, u(0)=u^{\prime}(0)=0, u^{\prime}(1)=\sum_{i=1}^{m-2} a_{i} u^{\prime}\left(\xi_{i}\right)$, where $D_{0^{+}}^{\alpha}$ denotes the standard RiemannLiouville fractional derivative, $f:[0,1] \times[0, \infty) \rightarrow[0, \infty)$ is a continuous function, $a_{i} \geq 0$ for $i=1,2, \ldots, m-2$, and $0<\xi_{1}<\xi_{2}<\cdots<\xi_{m-2}<1$. Our analysis relies on a fixed point theorem in partially ordered sets. Some examples are also presented to illustrate the main results.
\end{abstract}

\section{Introduction}

Many papers and books on fractional differential equations have appeared recently. Most of them are devoted to the solvability of the linear fractional equation in terms of a special function (see, e.g., [1,2]) and to problems of analyticity in the complex domain [3]. Moreover, Delbosco and Rodino [4] considered the existence of a solution for the nonlinear fractional differential equation

$$
D_{0^{+}}^{\alpha} u=f(t, u), \quad 0<\alpha<1,
$$

where $f:[0, a] \times \mathbb{R} \rightarrow \mathbb{R}, 0<a \leq+\infty$, is a given continuous function in $(0, a) \times \mathbb{R}$. They obtained existence results by using the Schauder fixed point theorem and the Banach contraction principle. 
Recently, El-Shahed [5] considered the following nonlinear fractional boundary value problem:

$$
\begin{gathered}
D_{0^{+}}^{\alpha} u+\lambda a(t) f(t, u(t))=0, \quad 0<t<1,2<\alpha \leq 3, \\
u(0)=u^{\prime}(0)=u^{\prime}(1)=0 .
\end{gathered}
$$

They used the Krasnoselskii's fixed point theorem on cone expansion and compression to show the existence and nonexistence of positive solutions for the above fractional boundary value problem. problem:

In [6], Liang and Zhang considered the following nonlinear fractional boundary value

$$
\begin{gathered}
D_{0^{+}}^{\alpha} u(t)+f(t, u(t))=0, \quad 0<t<1,3<\alpha \leq 4, \\
u(0)=u^{\prime}(0)=u^{\prime \prime}(0)=u^{\prime \prime}(1)=0,
\end{gathered}
$$

and by means of lower and upper solution method and fixed point theorems, they obtained some results on the existence of positive solutions to the above boundary value problem.

The question of uniqueness of the solution is not treated in [6].

Recently, in [7] Caballero et al. studied the fractional boundary value problem appearing in [6] by using a fixed point theorem in partially ordered sets, and the authors obtained uniqueness of the solution.

In this paper we discuss the existence and uniqueness of a positive and nondecreasing solution for the following $m$-point nonlinear boundary value problem of fractional order:

$$
\begin{gathered}
D_{0^{+}}^{\alpha} u(t)+f(t, u(t))=0, \quad 0<t<1,2<\alpha \leq 3, \\
u(0)=u^{\prime}(0)=0, \quad u^{\prime}(1)=\sum_{i=1}^{m-2} a_{i} u^{\prime}\left(\xi_{i}\right),
\end{gathered}
$$

where $a_{i} \geq 0$ for $i=1,2, \ldots, m-2,0<\xi_{1}<\xi_{2}<\cdots<\xi_{m-2}<1$, and, moreover, $0<\sum_{i=1}^{m-2} a_{i} \xi_{i}^{\alpha-2}<$ 1.

Recently, this problem has been studied in $[8,9]$. In [8] the author studies the existence and multiplicity of positive solutions for problem (1.4), and he uses Krasnoselskii and Leggett-Williams fixed point theorems. The question of uniqueness and monotonicity of the solution is not treated. In [9], the authors investigate the existence and uniqueness of positive and nondecreasing solutions for problem (1.4), and the main tools in this paper are a fixed point theorem in partially ordered sets and the lower and upper solution method.

Our study is based on a different fixed point theorem in partially ordered sets than the one used in [9]. $[8,9]$.

Our main interest in this paper is to give an alternative answer to the main results of

Existence of fixed points in partially ordered sets has been considered recently in [1013], among others. 
For existence theorems for fractional differential equations and applications, we refer to the survey [14]. Concerning the definitions and basic properties, we refer the reader to [15].

\section{Preliminaries and Previous Results}

For the convenience of the reader, we present here some definitions, lemmas, and results that will be used in the proofs of our main results.

Definition 2.1. The Riemann-Liouville fractional integral of order $\alpha>0$ of a function $f$ : $(0, \infty) \rightarrow \mathbb{R}$ is given by

$$
I_{0_{+}}^{\alpha} f(t)=\frac{1}{\Gamma(\alpha)} \int_{0}^{t}(t-s)^{\alpha-1} f(s) d s
$$

provided that the right-hand side is pointwise defined on $(0, \infty)$ and where $\Gamma(\alpha)$ denotes the Euler gamma function given by

$$
\Gamma(\alpha)=\int_{0}^{+\infty} t^{\alpha-1} e^{-t} d t, \quad \alpha>0 .
$$

Definition 2.2. The Riemann-Liouville fractional derivative of order $\alpha>0$ of a function $f$ : $(0, \infty) \rightarrow \mathbb{R}$ is defined by

$$
D_{0^{+}}^{\alpha} f(t)=\frac{1}{\Gamma(n-\alpha)}\left(\frac{d}{d t}\right)^{n} \int_{0}^{t} \frac{f(t)}{(t-s)^{\alpha-n+1}} d s,
$$

where $n=[\alpha]+1$ and $[\alpha]$ denote the integer part of $\alpha$.

The following two lemmas can be found in [16, 17].

Lemma 2.3. Let $\alpha>0$ and $u \in C(0,1) \cap L^{1}(0,1)$. Then the fractional differential equation

$$
D_{0^{+}}^{\alpha} u(t)=0
$$

has

$$
u(t)=c_{1} t^{\alpha-1}+c_{2} t^{\alpha-2}+\cdots+c_{n} t^{\alpha-n}, \quad c_{i} \in \mathbb{R}(i=1,2, \ldots, n), n=[\alpha]+1,
$$

as unique solutions.

Lemma 2.4. Assume that $u \in C(0,1) \cap L^{1}(0,1)$ with a fractional derivative of order $\alpha>0$ that belongs to $C(0,1) \cap L^{1}(0,1)$. Then

$$
I_{0_{+}}^{\alpha} D_{0^{+}}^{\alpha} u(t)=u(t)+c_{1} t^{\alpha-1}+c_{2} t^{\alpha-2}+\cdots+c_{n} t^{\alpha-n},
$$

for some $c_{i} \in \mathbb{R}(i=1,2, \ldots, n)$ and $n=[\alpha]+1$. 
Using Lemma 2.4, in [8] the following result is proved.

Lemma 2.5. Given $f \in C[0,1]$, then the unique solution of

$$
\begin{gathered}
D_{0^{+}}^{\alpha} u(t)+f(t)=0, \quad 0<t<1,2<\alpha \leq 3, \\
u(0)=u^{\prime}(0)=0, \quad u^{\prime}(1)=\sum_{i=1}^{m-2} a_{i} u^{\prime}\left(\xi_{i}\right)
\end{gathered}
$$

is

$$
u(t)=\int_{0}^{1} G(t, s) f(s) d s,
$$

where Green's function $G(t, s)$ is given by

$$
G(t, s)=G_{1}(t, s)+G_{2}(t, s),
$$

taking

$$
\begin{aligned}
& G_{1}(t, s)= \begin{cases}\frac{(1-s)^{\alpha-2} t^{\alpha-1}-(t-s)^{\alpha-1}}{\Gamma(\alpha)}, & 0 \leq s \leq t \leq 1, \\
\frac{(1-s)^{\alpha-2} t^{\alpha-1}}{\Gamma(\alpha)}, & 0 \leq t \leq s \leq 1,\end{cases} \\
& G_{2}(t, s)=\frac{1}{(1-\rho) \Gamma(\alpha)}\left(\rho(1-s)^{\alpha-2}-\sum_{i=1}^{m-2} a_{i}\left(\xi_{i}-s\right)^{\alpha-2} \chi E_{i}(s)\right) t^{\alpha-1},
\end{aligned}
$$

where $\rho=\sum_{i=1}^{m-2} a_{i} \xi_{i}^{\alpha-2}$ and $\chi_{E_{i}}$ denotes the characteristic function of the set $E_{i}=\left[0, \xi_{i}\right]$ for $i=$ $1,2, \ldots, m-2$.

Remark 2.6. Notice that Lemma 3 appears in [8] under assumption $2<\alpha<3$. In [9] the authors prove this result in the same way for $2<\alpha \leq 3$.

The following result is proved in $[8,9]$.

Lemma 2.7. Under the assumption $a_{i} \geq 0$ for $i=1,2, \ldots, m-2,0<\xi_{1}<\xi_{2}<\cdots<\xi_{m-2}<1$ and $1-\rho>0$ where $\rho=\sum_{i=1}^{m-2} a_{i} \xi_{i}^{\alpha-2}$, the Green's function appearing in Lemma 2.5 satisfies $G(t, s) \geq 0$.

Remark 2.8. It is easily checked that $G(t, s)$ is a continuous function on $[0,1] \times[0,1]$. 
The following lemmas appear in [9].

Lemma 2.9. The function $G_{1}(t, s)$ appearing in Lemma 2.5 is strictly increasing in the first variable.

Lemma 2.10. The function $G(t, s)$ appearing in Lemma 2.5 satisfies

$$
\max _{0 \leq t \leq 1} \int_{0}^{1} G(t, s) d s=\frac{1}{(\alpha-1) \Gamma(\alpha)}\left[\frac{1}{\alpha}+\frac{1}{1-\varrho}\left(\sum_{i=1}^{m-2} a_{i} \xi_{i}^{\alpha-2}\left(1-\xi_{i}\right)\right)\right] .
$$

For convenience, we will denote by $L$ the constant

$$
L=\frac{1}{(\alpha-1) \Gamma(\alpha)}\left[\frac{1}{\alpha}+\frac{1}{1-\varrho}\left(\sum_{i=1}^{m-2} a_{i} \xi_{i}^{\alpha-2}\left(1-\xi_{i}\right)\right)\right]
$$

In the sequel we present the fixed point theorem we will use later and which appears in [18].

Firstly, we need to introduce the following class of functions. By $\mathcal{S}$ we denote the class of those functions $\beta:[0, \infty) \rightarrow[0,1)$ satisfying $\beta\left(t_{n}\right) \rightarrow 1$ implies $t_{n} \rightarrow 0$.

Theorem 2.11 (see [18]). Let $(X, \leq)$ be a partially ordered set, and suppose that there exists a metric $d$ in $X$ such that $(X, d)$ is a complete metric space. Let $T: X \rightarrow X$ be a nondecreasing mapping such that there exists an element $x_{0} \in X$ with $x_{0} \leq T x_{0}$.

Suppose that there exists $\beta \in \mathcal{S}$ such that

$$
d(T x, T y) \leq \beta(d(x, y)) d(x, y),
$$

for $x, y \in X$ with $y \geq x$.

Assume that either $T$ is continuous or $\mathrm{X}$ is such that

if $\left(x_{n}\right)$ is a nondecreasing sequence with $x_{n} \longrightarrow x$ in $X$, then $x_{n} \leq x, \forall n \in \mathbb{N}$.

Besides, if

$$
\text { for } x, y \in X \text { there exists } z \in X \text { which is comparable to } x \text { and } y \text {, }
$$

then $T$ has a unique fixed point.

In our considerations, we will work in the space $C[0,1]=\{x:[0,1] \rightarrow \mathbb{R}$, continuous $\}$, with the standard distance given by $d(x, y)=\max _{0 \leq t \leq 1}\{|x(t)-y(t)|\}$ for $x, y \in C[0,1]$.

Notice that this space can be equipped with a partial order given by

$$
x \leq y \Longleftrightarrow x(t) \leq y(t),
$$

for $x, y \in C[0,1]$ and $t \in[0,1]$.

In $[12]$ it is proved that $(C[0,1], \leq)$ with the above-mentioned distance satisfies condition (2.14) of Theorem 2.11. Moreover, for $x, y \in C[0,1]$, as the function $\max \{x, y\}$ is continuous in $[0,1],(C[0,1], \leq)$ satisfies condition $(2.15)$ of Theorem 2.11 . 


\section{Main Result}

Our starting point in this section is to present the class of functions $\mathbb{A}$ which we use later. By $A$ we will denote the class of those functions $\phi:[0, \infty) \rightarrow[0, \infty)$ satisfying the following conditions:

(C1) $\phi$ is nondecreasing;

(C2) For any $x>0, \phi(x)<x$;

(C3) $\phi(x) / x \in \mathcal{S}$, where $\mathcal{S}$ is the class of functions appearing in Section 2. $\ln (1+x)$

Examples of functions in $\mathcal{A}$ are $\phi(x)=\mu x$ with $0 \leq \mu<1, \phi(x)=x / 1+x$ and $\phi(x)=$ In what follows, we formulate our main result.

Theorem 3.1. Suppose that the following assumptions are satisfied:

(a) $f:[0,1] \times[0, \infty) \rightarrow[0, \infty)$ is continuous;

(b) $f(t, x)$ is nondecreasing with respect to the second variable for each $t \in[0,1]$;

(c) there exists $0<\lambda \leq 1 / L$ and $\phi \in \mathcal{A}$ such that

$$
f(t, y)-f(t, x) \leq \lambda \phi(y-x)
$$

for $x, y \in[0, \infty)$ with $y \geq x$ and $t \in[0,1]$.

Then problem (1.4) has a unique nonnegative and nondecreasing solution.

Proof. Consider the cone $P=\{u \in C[0,1]: u \geq 0\}$.

Notice that, as $P$ is a closed set of $C[0,1], P$ is a complete metric space with the distance given by $d(x, y)=\max _{0 \leq t \leq 1}\{|x(t)-y(t)|\}$ satisfying conditions (2.14) and (2.15) of Theorem 2.11.

Now, for $u \in P$ we define the operator $T$ by

$$
(T u)(t)=\int_{0}^{1} G(t, s) f(s, u(s)) d s,
$$

where $G(t, s)$ is Green's function defined in Section 2.

By Lemma 2.7, Remark 2.8, and (a), it is clear that $T$ applies the cone $P$ into itself.

Now, we will check that assumptions in Theorem 2.11 are satisfied.

Firstly, the operator $T$ is nondecreasing.

In fact, by assumption (b), for $u \geq v$ we have

$$
(T u)(t)=\int_{0}^{1} G(t, s) f(s, u(s)) d s \geq \int_{0}^{1} G(t, s) f(s, v(s)) d s=(T v)(t)
$$


On the other hand, for $u \geq v$ and $u \neq v$ and taking into account our hypotheses, we can obtain

$$
\begin{aligned}
d(T u, T v) & =\max _{0 \leq t \leq 1}\{|(T u)(t)-(T v)(t)|\} \\
& =\max _{0 \leq t \leq 1}[(T u)(t)-(T v)(t)] \\
& =\max _{0 \leq t \leq 1}\left[\int_{0}^{1} G(t, s)(f(s, u(s))-f(s, v(s))) d s\right] \\
& \leq \max _{0 \leq t \leq 1}\left[\int_{0}^{1} G(t, s) \lambda \phi(u(s)-v(s)) d s\right] .
\end{aligned}
$$

Since $\phi$ is not nondecreasing and taking into account Lemma 2.10 and assumption (c), we get

$$
\begin{aligned}
d(T u, T v) & \leq \lambda \phi(\|u-v\|) \max _{0 \leq t \leq 1} \int_{0}^{1} G(t, s) d s \\
& =\lambda \phi(d(u, v)) L \leq \phi(d(u, v))=\frac{\phi(d(u, v))}{d(u, v)} d(u, v) .
\end{aligned}
$$

Thus, for $u \geq v$ and $u \neq v$,

$$
d(T u, T v) \leq \beta(d(u, v)) d(u, v)
$$

where $\beta(x)=\phi(x) / x \in \mathcal{S}$.

Obviously, the last inequality is satisfied for $u=v$.

Thus condition (2.13) in Theorem 2.11 holds with $\beta(x)=\phi(x) / x$. Moreover, since $f$ and $G$ are nonnegative functions,

$$
(T 0)(t)=\int_{0}^{1} G(t, s) f(s, 0) d s \geq 0 .
$$

Finally, Theorem 2.11 tells us that problem (1.4) has a unique nonnegative solution $u(t)$.

In what follows, we will prove that the unique nonnegative solution $u(t)$ for problem (1.4) is nondecreasing.

In fact, by Lemmas 2.5 and 2.9 it is easily seen that $G(t, s)$ is strictly increasing and, since $u(t)$ is a fixed point of the operator $T$, we have

$$
u(t)=\int_{0}^{1} G(t, s) f(s, u(s)) d s
$$

These facts and the nonnegative character of $f$ give us that $u(t)$ is nondecreasing.

Now, we present a sufficient condition for the existence and uniqueness of a positive and strictly increasing solution for problem (1.4) (positive solution means a solution 
satisfying $x(t)>0$ for $t \in(0,1))$. The proof of this fact is similar to the proof of Theorem 3.6 of [18]. We present it for completeness.

Theorem 3.2. Under assumptions of Theorem 3.1 and adding the following assumption

(d) $f\left(t_{0}, 0\right) \neq 0$ for certain $t_{0} \in[0,1]$, one obtains existence and uniqueness of a positive and strictly increasing solution for problem (1.4).

Proof. Consider the nonnegative solution $x(t)$ for Problem (1.4) whose existence is guaranteed by Theorem 3.1.

Notice that $x(t)$ satisfies

$$
x(t)=\int_{0}^{1} G(t, s) f(s, x(s)) d s
$$

Firstly, we will prove that $x(t)>0$ for $t \in(0,1)$.

In fact, in contrary case we can find $0<t^{*}<1$ such that $x\left(t^{*}\right)=0$. Consequently,

$$
x\left(t^{*}\right)=\int_{0}^{1} G\left(t^{*}, s\right) f(s, x(s)) d s=0
$$

As $x \geq 0, G(t, s) \geq 0$, and $f$ is nondecreasing with respect to the second variable (assumption (b)), from the last expression we can get

$$
0=x\left(t^{*}\right)=\int_{0}^{1} G\left(t^{*}, s\right) f(s, x(s)) d s \geq \int_{0}^{1} G\left(t^{*}, s\right) f(s, 0) d s \geq 0
$$

and, thus,

$$
\int_{0}^{1} G\left(t^{*}, s\right) f(s, 0) d s=0
$$

This fact and the nonnegative character of the functions $G\left(t^{*}, s\right)$ and $f(t, 0)$ imply

$$
G\left(t^{*}, s\right) f(s, 0)=0 \quad \text { a.e. }(s) \text {. }
$$

By Lemma 2.9, and since $G(0, s)=0$ and $G\left(t^{*}, s\right)>0$ for any $s \in[0,1]$, we have

$$
f(s, 0)=0 \quad \text { a.e. }(s) \text {. }
$$

On the other hand, assumption (d) gives us that $f\left(t_{0}, 0\right) \neq 0$ for certain $t_{0} \in[0,1]$ and, thus, $f\left(t_{0}, 0\right)>0$.

This fact and the continuity of $f$ imply the existence of a set $A \subset[0,1]$ with $t_{0} \in A$ and $\mu(A)>0$, where $\mu$ is the Lebesgue measure, such that $f(t, 0)>0$ for any $t \in A$.

This contradicts (3.14). 
Therefore, $x(t)>0$ for $t \in(0,1)$.

In the sequel, we will show that $x(t)$ is strictly increasing.

In fact, since $G(0, s)=0$, we have

$$
x(0)=\int_{0}^{1} G(0, s) f(s, x(s)) d s=0 .
$$

Now, we take $t_{1}, t_{2} \in[0,1]$ with $t_{1}<t_{2}$.

We can consider two cases.

Case $1(t=0)$. Suppose that $x\left(t_{2}\right)=0$.

Using a similar argument similar o the one used in the proof of the positive character of $x(t)$, we obtain a contradiction.

Case $2\left(t_{1}>0\right)$. Suppose that $x\left(t_{1}\right)=x\left(t_{2}\right)$.

In this case, we have

$$
\int_{0}^{1}\left(G\left(t_{1}, s\right)-G\left(t_{2}, s\right)\right) f(s, x(s)) d s=0 .
$$

Since $G\left(t_{1}, s\right)-G\left(t_{2}, s\right)>0$ (Lemma 2.3), we get

$$
f(s, x(s))=0 \quad \text { a.e. }(s) \text {. }
$$

Again, the same reasoning that we use earlier gives us a contradiction.

Therefore, $x\left(t_{1}\right)<x\left(t_{2}\right)$.

This finished the proof.

Remark 3.3. In Theorem 3.2, the condition $f\left(t_{0}, 0\right) \neq 0$ for certain $t_{0} \in[0,1]$ seems to be a strong condition in order to obtain a positive solution for problem (1.4), but when the solution is unique, we will see that this condition is very adjusted one. In fact, suppose that problem (1.4) has a unique nonnegative solution $x(t)$, then we have

$$
f(t, 0)=0 \quad \text { for any } t \in[0,1] \text { iff } x(t) \equiv 0 .
$$

Indeed, if $f(t, 0)=0$ for any $t \in[0,1]$, then it is easily seen that the zero function is a solution for problem (1.4) and the uniqueness of solution gives us $x(t) \equiv 0$.

The reverse implication is obvious.

Remark 3.4. Notice that assumptions in Theorem 3.1 are invariant by nonnegative and continuous perturbations. More precisely, if $f(t, 0)=0$ for any $t \in[0,1]$ and $f$ satisfies 
conditions (a), (b), and (c) of Theorem 3.1, then $g(t, x)=a(t)+f(t, x)$, where $a:[0,1] \rightarrow$ $[0, \infty)$ continuously and $a \neq 0$, satisfies assumptions of Theorem 3.2, and this means that the boundary value problem

$$
\begin{gathered}
D_{0^{+}}^{\alpha} u(t)+g(t, u(t))=0, \quad 0<t<1,2<\alpha \leq 3, \\
u(0)=u^{\prime}(0)=0, \quad u^{\prime}(1)=\sum_{i=1}^{m-2} a_{i} u^{\prime}\left(\xi_{i}\right),
\end{gathered}
$$

with $a_{i} \geq 0$ for $i=1,2, \ldots, m-2,0<\xi_{1}<\xi_{2}<\cdots<\xi_{m-2}<1$, and $0<\sum_{i=1}^{m-2} a_{i} \xi_{i}^{\alpha-2}<, 1$ has a unique positive and strictly increasing solution.

In the sequel, we present an example which illustrates our results.

Example 3.5. Consider the following boundary value problem:

$$
\begin{gathered}
D_{0^{+}}^{5 / 2} u(t)+t+\frac{\lambda u(t)}{1+u(t)}=0, \quad 0<t<1, \lambda>0, \\
u(0)=u^{\prime}(0)=0, \quad u^{\prime}(1)=\left(\frac{1}{2}\right) u^{\prime}\left(\frac{1}{4}\right)+\left(\frac{1}{4}\right) u^{\prime}\left(\frac{1}{2}\right) .
\end{gathered}
$$

In this case, $\alpha=5 / 2, f(t, u)=t+(\lambda u /(1+u)), 0<\xi_{1}=1 / 4<\xi_{2}=1 / 2<1, a_{1}=1 / 2$, and $a_{2}=1 / 4$. Besides, $\varrho=(1 / 2)(1 / 2)+(1 / 4)(1 / \sqrt{2}) \simeq 0.4267767$, and

$$
L=\frac{1}{(3 / 2) \Gamma(5 / 2)}\left[\frac{2}{5}+\frac{1}{1-\rho}\left(\frac{1}{2}\left(\frac{1}{4}\right)^{1 / 2} \frac{3}{4}+\frac{1}{4}\left(\frac{1}{2}\right)^{1 / 2} \frac{1}{2}\right)\right] \simeq 0.44197
$$

and $1 / L=2.2626$.

It is easily seen that $f(t, u)$ satisfies condition (a) of Theorem 3.1.

Since $\partial f / \partial u=\lambda /(1+u)^{2}>0$ for $u \in[0, \infty), f(t, u)$ satisfies (b) of Theorem 3.1.

Moreover, for $u \geq v$ and $t \in[0,1]$, we have

$$
\begin{aligned}
f(t, u)-f(t, v) & =t+\frac{\lambda u}{1+u}-\lambda-\frac{t v}{1+v} \\
& =\lambda\left(\frac{u}{1+u}-\frac{v}{1+v}\right)=\lambda\left(\frac{u-v}{(1+u)(1+v)}\right) \\
& \leq \lambda \frac{u-v}{1+u-v}=\lambda \phi(u-v),
\end{aligned}
$$

where $\phi(x)=x / 1+x$. It is easily proved that $\phi$ belongs to the class $\mathcal{A}$. Since $f(t, 0)=t \neq 0$ for $t \neq 0$, Theorem 3.2 states that problem (3.20) has a unique positive and strictly increasing solution for $0<\lambda \leq 1 / L \simeq 2.2626$. 


\section{Some Remark}

In [9] the authors consider our problem (1.4) and they prove the following result.

Theorem 4.1. Problem (1.4) has a unique positive and strictly increasing with solution $u(t)$ if the following conditions are satisfied:

(1) $f:[0,1] \times[0, \infty) \rightarrow[0, \infty)$ is continuous and nondecreasing with respect to the second variable and $f(t, u(t)) \neq 0$ for $t \in Z \subset[0,1]$ with $\mu(Z)>0$ ( $\mu$ denotes the Lebesgue measure),

(2) there exists $0<\lambda<1 / L$ such that for $u, v \in[0, \infty)$ with $u \geq v$ and $t \in[0,1]$

$$
f(t, u)-f(t, v) \leq \lambda \ln (u-v+1) .
$$

The main tool used by the authors in [9] for the proof of Theorem 4.1 is a fixed point theorem in partially ordered sets which appears in [19]. This fixed point theorem uses the following class of functions $\mathcal{M}$.

By $\mathcal{M}$ we denote the class of functions $\phi:[0, \infty) \rightarrow[0, \infty)$ continuously such that if $\varphi(x)=x-\phi(x)$, then the following conditions are satisfied:

(1) $\varphi:[0, \infty) \rightarrow[0, \infty)$, and it is nondecreasing;

(2) $\varphi(0)=0$;

(3) $\varphi$ is positive in $(0, \infty)$.

The same proof used by the authors in [9] gives us that the conclusion of Theorem 4.1 is true if we replace condition 2 of Theorem 4.1 by the following:

(2') there exists $0<\lambda<1 / L$ such that for $u, v \in[0, \infty)$ with $u \geq v$ and $t \in[0,1]$

$$
f(t, u)-f(t, v) \leq \lambda \phi(u-v),
$$

where $\phi \in \mathcal{M}$.

In what follows we will prove that the classes of functions $\mathcal{A}$ (see Section 3 ) and $\mathcal{M}$ are not comparable.

Example 4.2. This example appears in [10].

Consider the function $\phi:[0, \infty) \rightarrow[0, \infty)$ defined by

$$
\phi(t)= \begin{cases}0, & 0 \leq t \leq 2, \\ 2 t-4, & 2<t \leq 3, \\ \left(\frac{2}{3}\right) t, & 3<t .\end{cases}
$$

It is easily proved that $\phi \in \mathcal{A}$.

On the other hand, it is easily seen that $\varphi(t)=t-\phi(t)$ is not increasing, and, consequently, $\phi \notin \mathcal{M}$. 
Example 4.3. Consider the function $\phi:[0, \infty) \rightarrow[0, \infty)$ given by $\phi(x)=x-\arctan x$.

It is easily proved that $\phi \in \mathcal{M}$.

On the other hand, since $\beta(x)=\phi(x) / x=1-(\arctan x) / x$ and $\beta\left(t_{n}\right) \rightarrow 1$ when $t_{n} \rightarrow \infty$, we have that $\phi \notin \mathcal{A}$.

Examples 4.2 and 4.3 tell us that the results of this paper cover cases which cannot be treated by Theorem 4 which appears in [9] and vice versa.

In [9] the following result is also proved ([9, Theorem 5.1]).

Theorem 4.4. Problem (1.4) has a positive solution $u(t)$ if the following conditions are satisfied:

$\left(H_{f}\right) f(t, u) \in C([0,1] \times[0, \infty),[0, \infty))$ is nondecreasing relative to $u, f(t, \gamma(t)) \not \equiv 0$ for $t \in$ $(0,1)$, and there exists a positive constant $\mu<1$ such that

$$
K^{\mu} f(t, u) \leq f(t, K u)
$$

for $K \in[0,1]$, where

$$
\begin{aligned}
\gamma(t)=\int_{0}^{1} G(t, s) d s+\frac{1}{(1-\rho) \Gamma(\alpha)}[ & \int_{0}^{1} \rho(1-s)^{\alpha-2} d s \\
& \left.-\sum_{i=1}^{m-2} a_{i} \int_{0}^{1}\left(\xi_{i}-s\right)^{\alpha-2} X_{E_{i}}(s) d s\right] t^{\alpha-1} .
\end{aligned}
$$

In the sequel, we present an example which can be treated by Theorem 3.2, and it cannot be studied by Theorem 4.4.

Example 4.5. Consider the fractional boundary value problem

$$
\begin{gathered}
D_{0^{+}}^{5 / 2} u(t)+\left(t^{2}+1\right)(\lambda u(t)+c)=0, \quad 0<t<1, c>0,0<\lambda<1, \\
u(0)=u^{\prime}(0)=0, \quad u^{\prime}(1)=\left(\frac{1}{2}\right) u^{\prime}\left(\frac{1}{4}\right)+\left(\frac{1}{4}\right) u^{\prime}\left(\frac{1}{2}\right) .
\end{gathered}
$$

In this case, $\alpha=5 / 2, f(t, u)=\left(t^{2}+1\right)(\lambda u+c), 0<\xi_{1}=1 / 4<\xi_{2}=1 / 2<1, a_{1}=1 / 2$ and $a_{2}=1 / 4$. Besides, $\varrho=(1 / 2)(1 / 4)^{1 / 2}+(1 / 4)(1 / 2)^{1 / 2} \simeq 0.4267767$.

It is easily seen that $f(t, u)$ satisfies condition (a) of Theorem 3.1. Since $\partial f / \partial u=\lambda\left(t^{2}+\right.$ 1) $>0, f(t, u)$ satisfies $(b)$ of Theorem 3.1.

Moreover, for $u \geq v$ and $t \in[0,1]$, we have

$$
f(t, u)-f(t, v)=\lambda\left(t^{2}+1\right)(u-v) \leq 2 \lambda(u-v)=2 \phi(u-v),
$$

where $\phi(x)=\lambda x$. It is easily checked that $\phi \in \mathcal{A}$.

Since $f(t, 0)=c\left(t^{2}+1\right) \neq 0$ for $t \neq 0$ and, as $2 \leq(1 / L)=2.2626$ (see Example 3.5), Theorem 3.2 gives us existence and uniqueness of a positive and strictly increasing solution for Problem (4.6). 
On the other hand, we will show that condition $\left(H_{f}\right)$ appearing in Theorem 4.4 is not satisfied.

In fact, suppose that there exists $0<\mu<1$ such that

$$
K^{\mu} f(t, u) \leq f(t, K u)
$$

for any $K \in[0,1]$.

Since $f(t, u)=\left(t^{2}+1\right)(\lambda u+c)$ with $0<\lambda<1$ and $c>0$, we have

$$
K^{\mu} \leq \frac{f(t, K u)}{f(t, u)}=\frac{\left(t^{2}+1\right)(\rho K u+c)}{\left(t^{2}+1\right)(\rho u+c)}=\frac{\rho K u+c}{\rho u+c} .
$$

Taking limit when $u \rightarrow \infty$ in the last expression, we get $K^{\mu} \leq K$, which is false, since $0<\mu<1$ and the function $h(\alpha)=K^{\alpha}$ is decreasing when $0<K<1$.

Therefore, Problem (4.6) can be covered by Theorem 3.2, and it cannot be treated using Theorem 4.4 .

In $[8]$, the author proves the following result.

Theorem 4.6. Assume that

(H1) $f:[0,1] \times[0, \infty) \rightarrow[0, \infty)$ is continuous,

(H2) $a_{i} \geq 0$ for $i=1,2, \ldots, m-2,0<\xi_{1}<\xi_{2}<\cdots<\xi_{m-2}<1$ and $\rho=\sum_{i=1}^{m-2} a_{i} \xi_{i}^{\alpha-2}$ with $\rho<1$.

In addition, suppose that one of the following two conditions holds:

(H3) $\lim _{u \rightarrow 0} \min _{t \in[0,1]} f(t, u) / u=\infty, \lim _{u \rightarrow \infty} \max _{t \in[0,1]} f(t, u) / u=0$,

(H4) $\lim _{u \rightarrow 0} \min _{t \in[0,1]} f(t, u) / u=0, \lim _{u \rightarrow \infty} \max _{t \in[0,1]} f(t, u) / u=\infty$.

Then Problem (1.4) has at least one positive solution.

In what follows we present an example which can be treated by our results and it cannot be studied by Theorem 4.6.

Example 4.7. Consider Problem (3.20) which appears in Example 3.5.

We prove that this example can be treated by Theorem 3.2 and we obtained a unique positive and strictly increasing solution for this problem when $0<\lambda<1 / L \simeq 2.2626$.

On the other hand, in this case, since $f(t, u)=t+(\lambda u /(1+u))$, we have

$$
\begin{gathered}
\min _{t \in[0,1]}\left(\frac{t+(\lambda u /(1+u))}{u}\right)=\frac{\lambda u /(1+u)}{u}=\frac{\lambda}{1+u^{\prime}} \\
\lim _{u \rightarrow 0} \min _{t \in[0,1]}\left(\frac{t+(\lambda u /(1+u))}{u}\right)=\lim _{u \rightarrow 0} \frac{\lambda}{1+u}=\lambda .
\end{gathered}
$$

Since $0<\lambda<\infty$, Theorem 4.6 cannot be used in this case. 
Example 4.8. Consider the following boundary value problem

$$
\begin{aligned}
& D_{0^{+}}^{5 / 2} u(t)+c+\lambda \arctan u(t)=0, \quad 0<t<1, \quad c>0, \quad \jmath>0, \\
& u(0)=u^{\prime}(0)=0, \quad u^{\prime}(1)=\left(\frac{1}{2}\right) u^{\prime}\left(\frac{1}{4}\right)+\left(\frac{1}{4}\right) u^{\prime}\left(\frac{1}{2}\right) .
\end{aligned}
$$

In this case, $\alpha=5 / 2, f(t, u)=c+\lambda \arctan u, 0<\xi_{1}=1 / 4<\xi_{2}=1 / 2<1, a_{1}=1 / 2$, and $a_{2}=1 / 4$. Besides, $\varrho=(1 / 2)(1 / 2)+(1 / 4)(1 / \sqrt{2}) \simeq 0.4267767$.

It is easily seen that $f(t, u)$ satisfies conditions (a) and (b) of Theorem 3.1.

Moreover, in [20] it is proved that if $u \geq v \geq 0$,

$$
\arctan u-\arctan v \leq \arctan (u-v) .
$$

Using this fact, for $u \geq v$ and $t \in[0,1]$, we have

$$
f(t, u)-f(t, v)=\lambda(\arctan u-\arctan v) \leq \lambda \arctan (u-v)=\lambda \phi(u-v),
$$

where $\phi(x)=\arctan x$.

In [20] it is proved that $\phi \in \mathscr{A}$.

Since $f(t, 0)=c \neq 0$, Theorem 3.2 gives us the existence and uniqueness of a positive and strictly increasing solution for Problem (4.11) when $0<\lambda \leq 1 / L \simeq 2.2626$.

On the other hand, since

$$
\begin{gathered}
\lim _{u \rightarrow 0} \min _{t \in[0,1]} \frac{f(t, u)}{u}=\lim _{u \rightarrow 0} \min _{t \in[0,1]} \frac{c+\lambda \arctan u}{u}=\lim _{u \rightarrow 0} \frac{c+\lambda \arctan u}{u}=\infty, \\
\lim _{u \rightarrow \infty} \max _{t \in[0,1]} \frac{f(t, u)}{u}=\lim _{u \rightarrow \infty} \frac{c+\lambda \arctan u}{u}=0,
\end{gathered}
$$

Problem (4.11) can be treated by Theorem 4.6, and we obtain the existence of at least one positive solution.

Our main contribution is that for $0<\lambda \leq 1 / L \simeq 2.2626$ we obtain uniqueness and strictly increasing character for the solution of Problem (4.11).

\section{Acknowledgment}

This paper was partially supported by the Ministerio de Educación y Ciencia, Project MTM $2007 / 65706$.

\section{References}

[1] L. M. B. C. Campos, "On the solution of some simple fractional differential equations," International Journal of Mathematics and Mathematical Sciences, vol. 13, no. 3, pp. 481-496, 1990.

[2] K. S. Miller and B. Ross, An Introduction to the Fractional Calculus and Fractional Differential Equations, John Wiley \& Sons, New York, NY, USA, 1993. 
[3] L. Yi and D. Shusen, "A class of analytic functions defined by fractional derivation," Journal of Mathematical Analysis and Applications, vol. 186, no. 2, pp. 504-513, 1994.

[4] D. Delbosco and L. Rodino, "Existence and uniqueness for a nonlinear fractional differential equation," Journal of Mathematical Analysis and Applications, vol. 204, no. 2, pp. 609-625, 1996.

[5] M. El-Shahed, "Positive solutions for boundary value problem of nonlinear fractional differential equation," Abstract and Applied Analysis, Article ID 10368, 8 pages, 2007.

[6] S. Liang and J. Zhang, "Positive solutions for boundary value problems of nonlinear fractional differential equation," Nonlinear Analysis, vol. 71, no. 11, pp. 5545-5550, 2009.

[7] J. Caballero, J. Harjani, and K. Sadarangani, "Existence and uniqueness of positive solution for a boundary value problem of fractional order," Abstract and Applied Analysis, Article ID 165641, 12 pages, 2011.

[8] W. Zhong, "Positive solutions for multipoint boundary value problem of fractional differential equations," Abstract and Applied Analysis, Article ID 601492, 15 pages, 2010.

[9] S. Liang and J. Zhang, "Existence anduniqueness of positive solutions to m-point boundary value problem for nonlinear fractional differential equation," Journal of Applied Mathematics and Computing. In press.

[10] A. Amini-Harandi and H. Emami, "A fixed point theorem for contraction type maps in partially ordered metric spaces and application to ordinary differential equations," Nonlinear Analysis, vol. 72, no. 5, pp. 2238-2242, 2010.

[11] J. Harjani and K. Sadarangani, "Fixed point theorems for weakly contractive mappings in partially ordered sets," Nonlinear Analysis, vol. 71, no. 7-8, pp. 3403-3410, 2009.

[12] J. J. Nieto and R. Rodríguez-López, "Contractive mapping theorems in partially ordered sets and applications to ordinary differential equations," Order, vol. 22, no. 3, pp. 223-239, 2005.

[13] D. O’Regan and A. Petruşel, "Fixed point theorems for generalized contractions in ordered metric spaces," Journal of Mathematical Analysis and Applications, vol. 341, no. 2, pp. 1241-1252, 2008.

[14] A. A. Kilbas and J. J. Trujillo, "Differential equations of fractional order: methods, results and problems. I," Applicable Analysis, vol. 78, no. 1-2, pp. 153-192, 2001

[15] S. G. Samko, A. A. Kilbas, and O. I. Marichev, Fractional Integrals and Derivatives. Theory and applications, Gordon and Breach Science Publishers, Yverdon, Switzerland, 1993.

[16] Z. Bai and H. Lü, "Positive solutions for boundary value problem of nonlinear fractional differential equation," Journal of Mathematical Analysis and Applications, vol. 311, no. 2, pp. 495-505, 2005.

[17] A. A. Kilbas, H. M. Srivastava, and J. J. Trujillo, Theory and Applications of Fractional Differential Equations, vol. 204 of North-Holland Mathematics Studies, Elsevier Science B.V., Amsterdam, The Netherlands, 2006.

[18] J. Caballero Mena, J. Harjani, and K. Sadarangani, "Existence and uniqueness of positive and nondecreasing solutions for a class of singular fractional boundary value problems," Boundary Value Problems, Article ID 421310, 10 pages, 2009.

[19] J. Harjani and K. Sadarangani, "Fixed point theorems for weakly contractive mappings in partially ordered sets," Nonlinear Analysis, vol. 71, no. 7-8, pp. 3403-3410, 2009.

[20] J. Caballero, J. Harjani, and K. Sadarangani, "Uniqueness of positive solutions for a class of fourthorder boundary value problems," Abstract and Applied Analysis, Article ID 543035, 13 pages, 2011. 


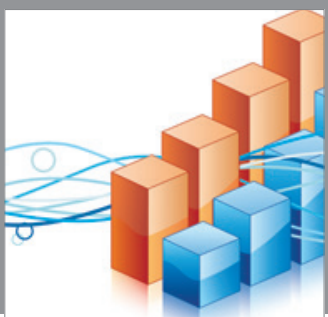

Advances in

Operations Research

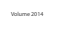

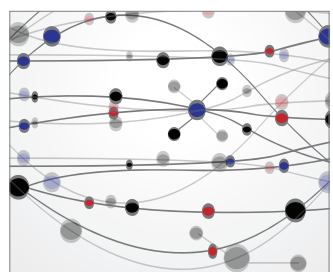

\section{The Scientific} World Journal
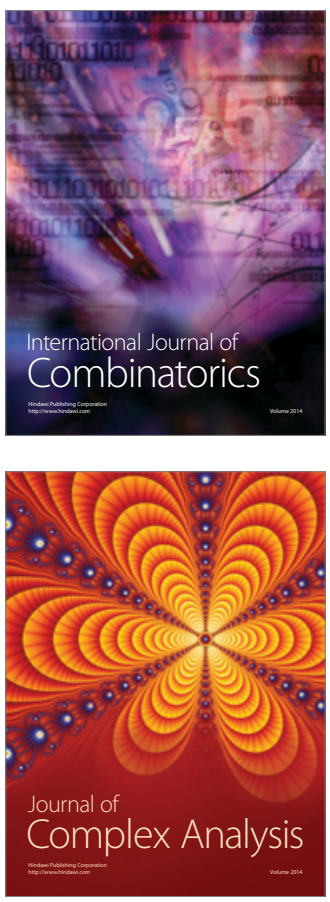

International Journal of

Mathematics and

Mathematical

Sciences
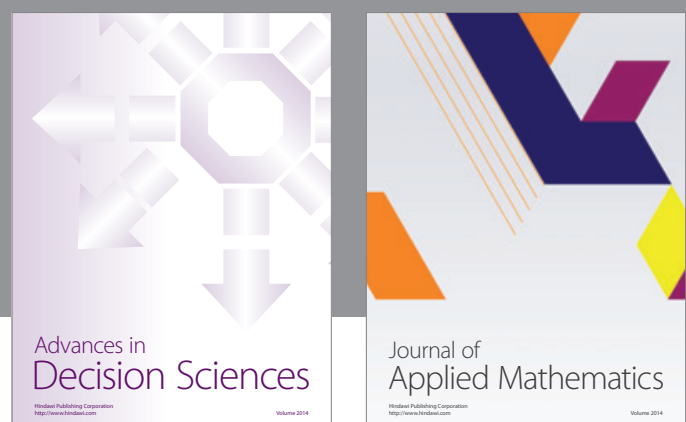

Journal of

Applied Mathematics
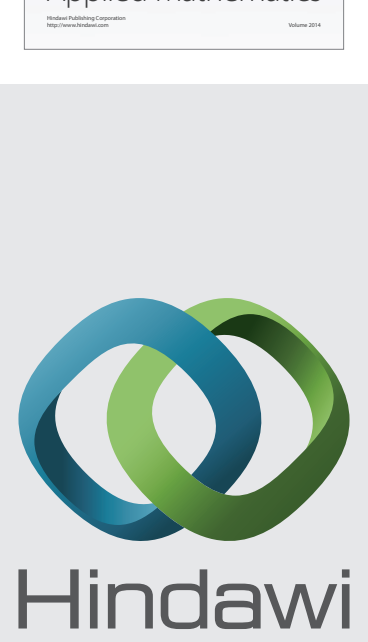

Submit your manuscripts at http://www.hindawi.com
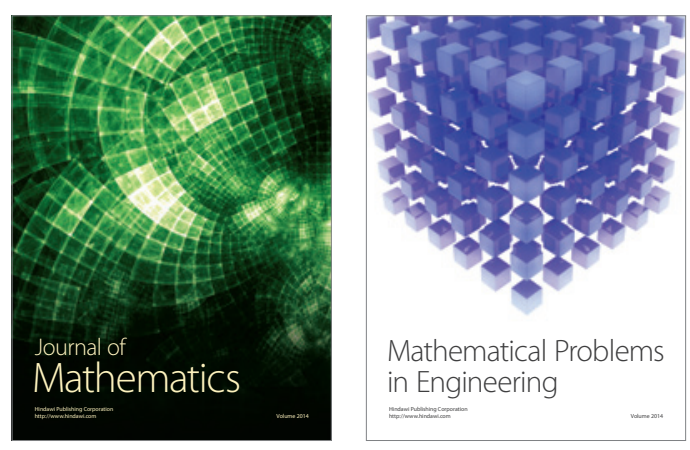

Mathematical Problems in Engineering
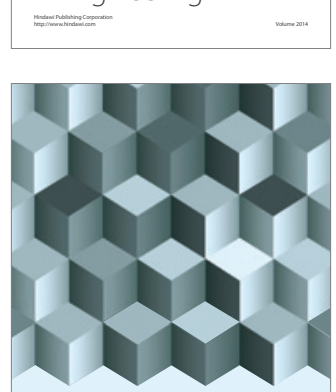

Journal of

Function Spaces
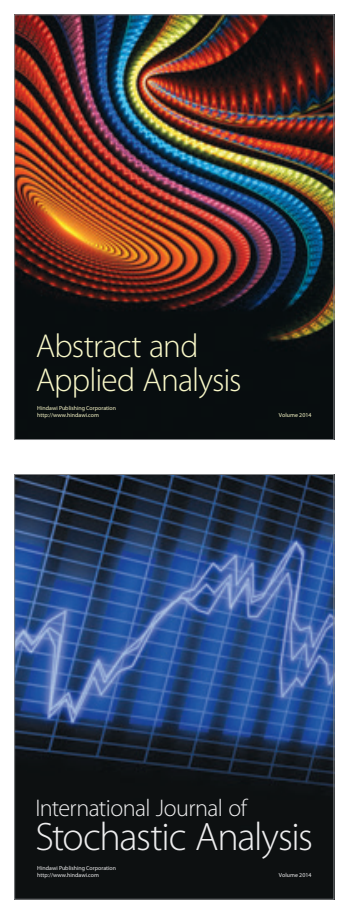

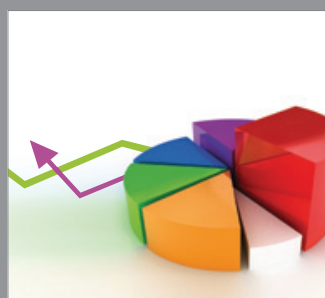

ournal of

Probability and Statistics

Promensencen
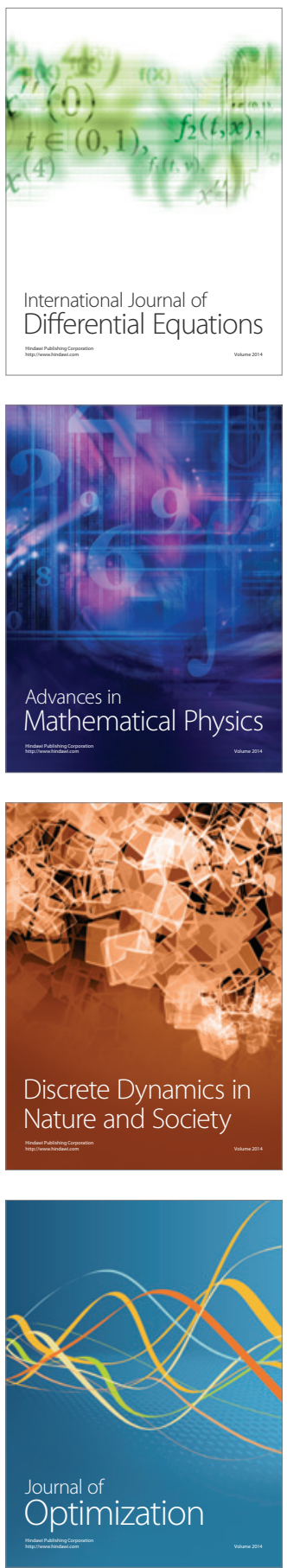